\title{
A state of the art regarding urban air quality prediction models
}

\author{
Cristiana Croitoru, ${ }^{l, *}$ and Ilinca Nastase \\ ${ }^{1}$ CAMBI Research Center, Technical University of Civil Engineering Bucharest, 021414 Bucharest, Romania
}

\begin{abstract}
Urban pollution represents an increasing risk to residents of urban regions, particularly in large, over-industrialized cities knowing that the traffic is responsible for more than $25 \%$ of air gaseous pollutants and dust particles. Air quality modelling plays an important role in addressing air pollution control and management approaches by providing guidelines for better and more efficient air quality forecasting, along with smart monitoring sensor networks. The advances in technology regarding simulations, forecasting and monitoring are part of the new smart cities which offers a healthy environment for their occupants.
\end{abstract}

\section{Introduction}

\subsection{Importance of indoor and exterior pollution}

Urban air pollution represents a growing risk to citizens, particularly in large, over-industrialized cities knowing that the traffic is responsible for more than $25 \%$ of air gaseous pollutants and dust particles. In Europe, an average of $80 \%$ of the population lives in urban areas [1]. The report of OECD [2] it is stated that air pollution is one of the most serious environmental risks. According to the World Health Organization (WHO) fine particulate matter is associated with a broad spectrum of acute and chronic illness, such as lung cancer, chronic obstructive pulmonary disease (COPD) and cardiovascular diseases. Worldwide, it is estimated to cause about $25 \%$ of lung cancer deaths, $8 \%$ of COPD deaths, and about $15 \%$ of ischaemic heart disease and stroke [3]. The economic costs of the derived effects were quantified in 2010 at 1.5 trillion euros [4].

The air pollutants can be divided into two groups based on the origin of the chemical composition and their physical states. According to WHO, the main emission sources of these pollutants are the use of fossil resources, the transportation vehicles, the power plants, and the production of oil and gas [5]. The primary type of air pollutants is concerning those that enter directly into the atmosphere, such as sulfur dioxide $\left(\mathrm{SO}_{2}\right)$, the nitrogen oxides $\left(\mathrm{NO}_{2}\right)$, and the hydrocarbons (HC). The secondary type of contaminants is concerning those that can be generated within the atmosphere by photochemical reactions of hydrolysis or oxidation, such as the ozone $\left(\mathrm{O}_{3}\right)$. Aerosols are considered an important source of pollution in urban areas [6, 7]. Aerosols (particulate matter-PM) include dust, gas emission, smoke, fog and fibers. PM are a complex mixture of chemical species, originating from a variety of sources, both anthropogenic (traffic, industrial activities and combustion processes in general) and natural (dust storms, forest fires, pollen, volcanic eruptions). They may be emitted as primary pollutants or formed as a secondary product through atmospheric processes from gaseous precursors. According to the latest report of the European Environmental Agency (EEA), in 2017 a large number of European cities displayed values above the admitted concentrations of the air quality standards for PM10 and PM2.5, with the majority of critical concentrations observed in Eastern Europe and the Balkan region [6]. For PM10 particles, the concentrations above the EU daily limit value were registered at $19 \%$ of the reporting stations in 20 of the 28 EU Member States [8]. A comprehensive study [9] reviews the basic evidence on the health effects of particulate matter. It addresses the major health risks in order to alert policy-makers so that more stringent strategies can be implemented to reduce air pollution and its health effects.

On the other hand, ozone can increase the incidence of asthma episodes, cause shortness of breath, aggravate lung diseases and can cause permanent lungs damage through long-term exposure. High ozone levels are linked to increases in hospitalizations, emergency room visits and premature death [10].

Considering the indoor pollution, besides the actual health problems, some other symptoms can be observed at the occupants affected, which cannot be linked directly to a specific pollutant. Starting with the late 1970 s, it was noted that nonspecific symptoms were reported by tenants in newly constructed buildings, but the term was established by the World Health Organization (WHO) in 1982 [11]. In 1984 the WHO issued a report suggesting that up to $30 \%$ of new and remodeled buildings may have excessive complaints about indoor air quality [12]. As stated in an article [13] from the prestigious medical journal, The Lancet, SBS is an increasingly frequent problem since it can be

\footnotetext{
* Corresponding author: cristiana.croitoru@utcb.ro
} 
uncomfortable, even disabling, and whole workplaces can be turned into non-functional.

Even though levels of particle matter and ground-level ozone pollution are significantly lower than in the past, levels still have a negative impact on human health. Both pollutants are the result of emissions from various sources and can travel long distances, affecting large regions.

\subsection{Importance of pollution prediction}

Air quality modelling plays an important role in addressing air pollution control and management approaches by providing guidelines for better and more efficient air quality forecasting[14]. As air pollution is a complex blend of contaminant components with considerable impact on human health, predicting air pollution concentrations appears as a priority for improving life quality [15]. It is demonstrated that the knowing how the pollution evolve helps in a better urban management with regards to inhabitants, allowing obtaining risk maps, especially when air quality indices are a tool for alertness, particularly important for vulnerable populations. The purpose of pollution modelling is to predict certain levels for certain situations in order to provide their citizens an opportunity to modify their personal habits to minimize exposure [16]. While substitute measures, such as distance to source of pollution, have been associated to large health effects [17], the problem of lowering the impact still exists. It is still needed to know how a certain concentration could evolve in certain environmental conditions and what effects will produce. The methods to evaluate the pollution dispersion utilize geographic information systems (GIS) to combine available geographic data with short-term monitoring information to develop exposure models capable of identifying small-area variations in pollution. Results from these models can then be overlapped on other georeferenced data, like health risks, in order to emphasise the exposure of people at their homes, work etc.

\section{Air pollution modelling}

Air pollution modelling is using mathematical approaches to explain or predict the way pollutants behave in a certain environment or time scale, even we talk about indoor or outdoor. Numerical models can be repeated indeterminately with strict parameters at relatively low costs comparing to physical modelling. Modelling helps evaluate the correlation between the pollutants sources and their effects on ambient air quality. Simulations can be used to run scenarios, to test concepts and to acknowledge environmental impact under different emission sources and levels, climate and conditions. Different methods and techniques exist, but the purpose is permanently the same - evaluate the pollutant impact over a given dimension (space, time) using an existing set of measured data. Although there are no clear differences among diverse categories, models might be classified after [18] into groups according to their physical (e.g. reduced-scale) or mathematical principles (e.g. box, Gaussian, regression, CFD) or their level of complexity (e.g. screening, semiempirical, numerical).

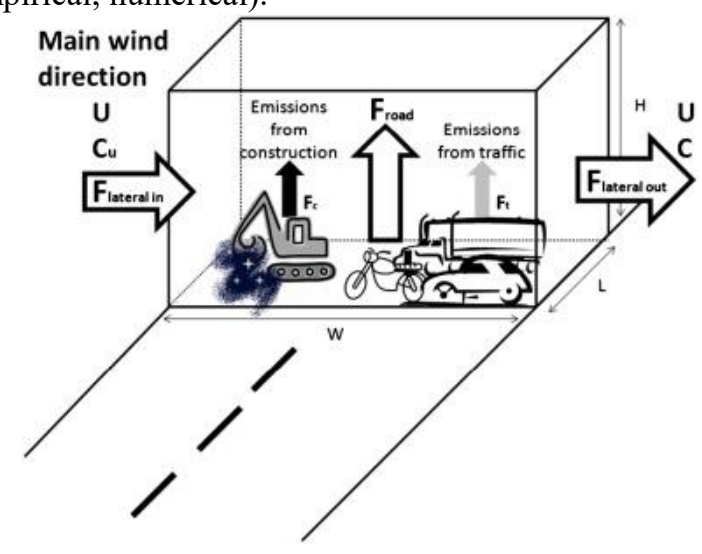

Fig. 1. Example of schematic diagram of the box model used to calculate PM10 emissions from the road construction phase of the road widening [19]

Chemical reactions between the pollutants can be integrated in these models, predicting also the effects generated by a certain critical situation with regards to secondary species which are not directly emitted as such, but form when other pollutants (primary pollutants) react in the atmosphere. Examples of a secondary pollutant include ozone, which is formed when hydrocarbons (HC) and nitrogen oxides (NOx) combine in the presence of sunlight. NO2 is formed as NO, reacts with oxygen in the air. Acid rain is formed when sulfur dioxide or nitrogen oxides react with water[20].

Moreover, urban air pollution simulation encounter problems with complex turbulent airflows like localscale topography (e.g. canyons, mountains, tall buildings, urban streets etc.), airborne particles and gases in the atmosphere, air circulation due to thermal effects or due to other sources etc. These problems can be resolved by specific simulations and the Computer Fluid Dynamics (CFD) methods and tools have become extensively used. CFD modelling is a specific term used to describe the quantitative and qualitative evaluation of systems involving fluid flow, heat transfer and associated phenomena (e.g. chemical reactions, diffusion etc.) using computer-based numerical methods. Among the first CFD studies related to pollution, we remark Svensson's[21], who was one of the firsts who started to use commercial CFD tools for various air-pollution problems. However, for outdoor air pollution other specific problems might appear and should be treated carefully. A substantial problem is the discrepancy of input and output boundary conditions for meteorological profiles taking into account the Coriolis forces, surface layer structure etc.[22]. Atmospheric pollution domain, CFD models are generally used when pollutant dispersion is highly influenced by the geometry of an urban area. Accurately describing the topography of a certain urban zone is very important. Chu et al.[23] even used geographic information system (GIS) software to obtain detailed coordinates and dimensions of the buildings in the interest area. Neofytou et al. [24] 
studied, using CFD methods, the formation of the vortices in the streets canyons due to the wind direction and the speed and the influence of the characteristics of the flow fields on the concentration distributions. Haghighat et al.[25] studied the impact of non-uniform urban surface temperature on pollution dispersion in urban areas, introducing also a radiation and a soil model to simulate the effect of heat storage. Other urban characteristics can be implemented to the model: in [26] Jeanjean et al. studied the effect of trees to disperse road traffic emissions at a city scale. The authors found that trees have a local beneficial impact on road traffic emissions by increasing turbulence and reducing ambient concentrations of road traffic emissions by $7 \%$ at pedestrian height. We may see that conditions imposed in models can vary on different aspects, but in the same time, a complex model will require important computation resources. The turbulence closure techniques used for the CFD models, when considering air pollution at urban level, two main types of turbulence models are used: Reynolds-averaged Navier-Stokes (RANS) and Large-Eddy Simulation (LES) [14] as the atmospheric turbulent flow in and above street canyons involves turbulent eddies on a variety of scales [27].
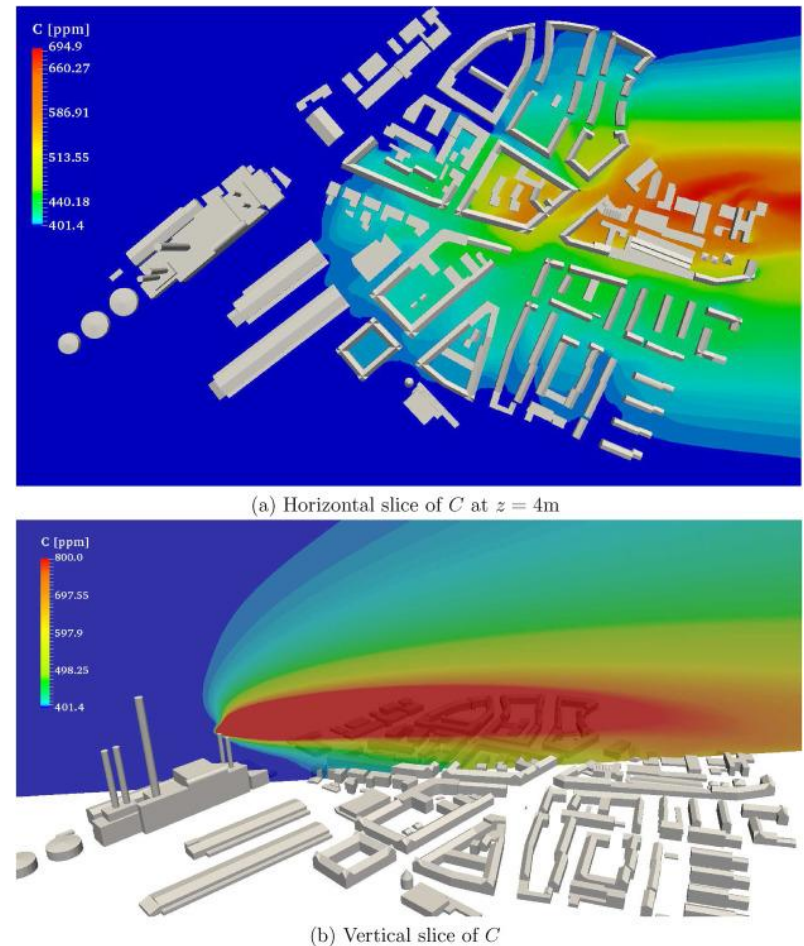

Fig. 4. Example of CFD modelling: images of the $\mathrm{CO}_{2}$ concentration[28]

An interesting alternative to these models are the neural network models [29, 30]. Artificial intelligence mechanisms are more and more used since they learn from real measured data and predict a certain parameter, e.g. artificial neural networks, Bayesian neural networks or adaptive neuro-fuzzy algorithms.

Most current air quality predicting models use direct, simple approaches like box models, Gaussian models or linear statistical models. Those models are easy to implement and allow a fast calculation and low computation resources. However, in general, they do not define the relations and non-linear relationship which govern the transport and behaviour of pollutants in the atmosphere[31]. To overcome these problems, artificial intelligence models have become popular in air quality forecasting and other atmospheric domains.

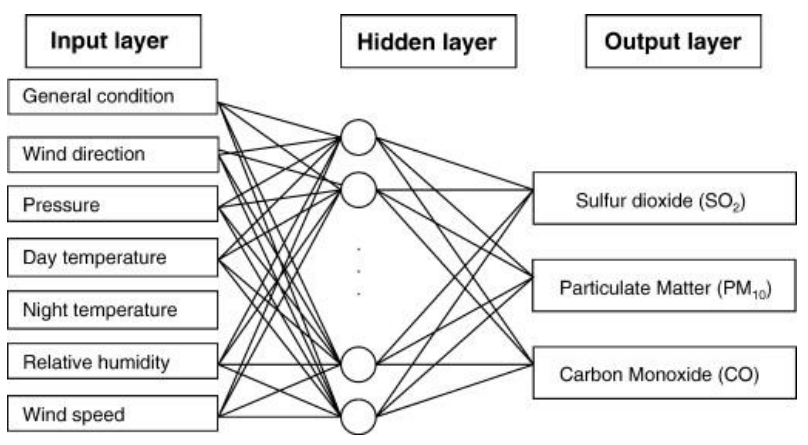

Fig. 3. Example of a neural network model[32]

Air quality management organisms worldwide use specific models which rely on mathematical and numerical techniques to simulate the physical and chemical processes of pollutants as they disperse and react in the atmosphere. The most commonly used air quality (AQ) models include: Dispersion Modelling estimates the concentration of pollutants at specified ground-level receptors surrounding an emissions source; Photochemical Modelling - used in regulatory or policy assessments by estimating pollutant concentrations and deposition of both inert and chemically reactive pollutants over large spatial scales; Receptor Modelling use the chemical and physical characteristics of gases and particles measured at source and receptor to quantify source contributions to receptor concentrations[33]. Different types of such models are analysed in a model evaluation study regarding the ozone level in Europe and Norths America [34], offering an opportunity to investigate methodologies for generating skilful ensembles of regional air quality models outputs. .

Air pollution is mostly addressed by national projects focusing mostly to tackle the health effects of ultrafine particles and air pollution due to combustion of fossil fuels [35]. On the other hand, most atmospheric models used in air quality management don't have a userfriendly interface that can synthesize the model results produced from different air pollutant emission inputs, and do not offer adequate data visualization for supporting policy making. To improve the efficiency of data synthesis, it is necessary to develop a tool to translate the massive amount of model data to policyrelevant visualization [36].

\section{Air pollution monitoring}

Field measurements can provide immediate data on pollutant concentration, air flow and pollutant dispersion, depending to the limitations of measurement techniques. Models which require learning data or those which require certain specifications are relying on the measured parameters. The restrictions encountered are often related to challenges to data interpretation, 
uncontrollable meteorological conditions, low spatial coverage, or typically high costs.

Pollution monitoring is a first step in resolving this problem, but we often remain at monitoring phase. Current sector-specific initiatives and priorities are mostly elaborated in isolation from each other - they lack consistency and sometimes counter-act each $\mathrm{x}$ should allow a cohesive solution for urban air pollution problems. Ideally, such policy decision support tools should be efficient, easy-to-use, and capable of estimating air quality changes in the quality of environment in real time for introducing given emission reduction scenarios for a particular city and/or relevant control actions. The literature survey indicates that pollution monitoring at urban scale has been approached in several projects, even with the dedicated purpose for policies implementation [37]. However, communities still need the link between the monitoring process and action taking.

Monitoring of urban pollutants is usually done through a network of sensors placed in specific areas. It also comprises the predicting step and data interpretation. The monitoring systems depend on the type of pollutant(s) or the target (local monitoring, district, city area etc.). Monitoring stations should be generally located near populated regions where pollutant exposure is the highest. Critical pollutants or with high risk, like ozone, have specific monitoring platforms, using certain types of technologies[38]. At global level it is also important to know the reference levels of pollutants in order to consider the urban levels. In a comprehensive review, Martin et al. [39] mentions the capabilities for satellite remote sensing of certain species(aerosols, tropospheric $\mathrm{O}_{3}$, tropospheric $\mathrm{NO}_{2}, \mathrm{CO}, \mathrm{HCHO}$, and $\mathrm{SO}_{2}$ ) in the boundary layer, along with physical processes affecting their accuracy and precision. The purpose is to achieve higher spatial resolution to resolve urban scales, facilitate validation and reduce cloud contamination that increases remote sensing error.

At the urban level, the monitoring networks usually transmit the information and different alert levels are issued. Different indices have been issued so far for air quality, from which we mention for example the Air Quality Health Index (AQHI) which is a public information tool designed in Canada to evaluate the impact of air quality on health.

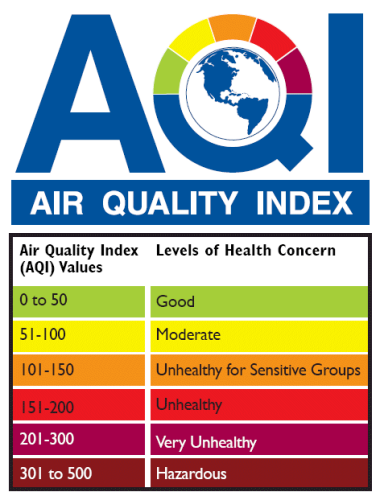

Fig. 5. Air Quality Index (AQI)calculated by EPA
A similar index is proposed by US Environmental Protection Agency: The Air Quality Index-AQI is an index for reporting daily air quality also with impact on citizens health. European Environment Agency is proposing the European Air Quality Index with values for different cities across Europe, but less data for Southeastern Europe.

Large, polluted cities have become more and more sensitive to air quality since pollution levels reach critical values. A heavy 16-day pollution episode occurred in Beijing was studied in [40] to reveal the combined effects of traffic restrictions and meteorology on urban air quality. Results indicated that additive functions of traffic restrictions, suitable relative humidity and temperature are more effective on concentration reduction only for some pollutants. More solutions are necessary to determine efficient ways for pollution control.

Urban networks are now equipped with wireless sensors, which transmit the data to dedicated servers where real time decision making takes place. The advances in technology have permitted the installation at large scale, monitoring large urban areas. Common architectures of information flow in wireless sensor networks is presented in figure 6 .

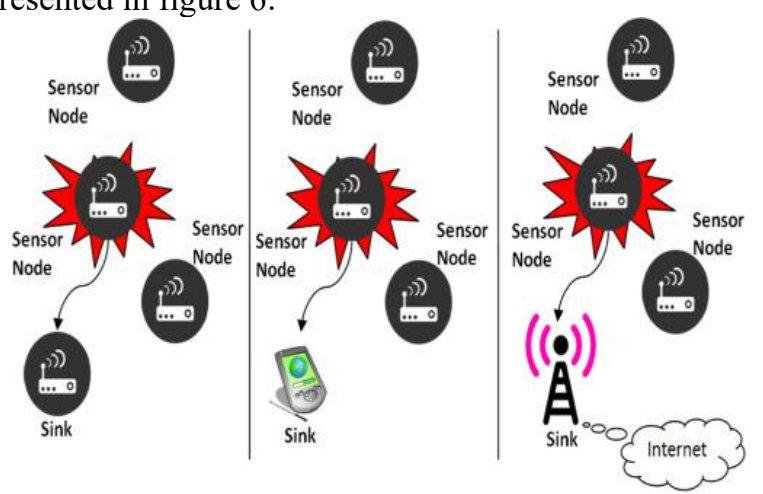

Fig. 6. Prospective architectural topologies of wireless sensor networks[41]

Recent studies reveal the importance of local-level pollution data, including human personal exposure to air pollutants. Connecting an array of wireless sensors to a server it is a simple and robust alternative of former urban sensor networks. The data can be transmitted via a General Packet Radio Service Modem (GPRS-Modem) and a Global Positioning System Module (GPS-Module), a simple and low cost solution.[42].

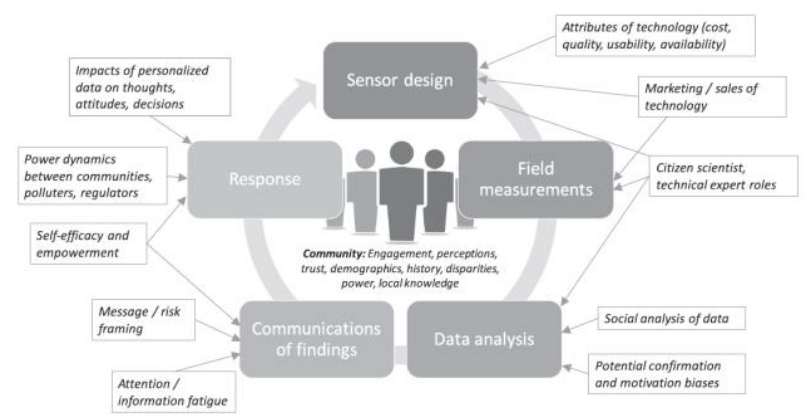

Fig. 7. Social sciences can contribute to the understanding of all elements of sensor design and use[43] 
Moreover, utilizing low-cost air quality sensors and the ordinary smartphones, end-users (simple citizens) can be more involved and are able to extract, analyse and share the local air pollution information [44, 45].

This the concept of "participatory sensing", which has a promising trend, especially when influenced by social context. In participatory sensing, individuals and groups engage in the data collection actively and the helps the public administration to make proper decisions[43, 46].

\section{Urban pollution- regulatory aspects and lack of consistency}

Pollution monitoring is a first step in resolving this problem, but we often remain at monitoring phase. Current sector-specific initiatives and priorities are mostly elaborated in segregation from each other - they lack of consistency and sometimes counter-act each other [47]. Large amount of collected data has not been integrated into suitable software applications which should allow a consistent solution for urban air pollution management. Ideally, such policy decision support tools should be efficient, easy-to-use and capable of estimating air quality changes in real time. The purpose is to introduce emission reduction scenarios previously considered for a particular region and/or relevant verification actions. In addition, the tools should offer intuitive policy-related visualizations [36].

Studies worldwide are showing that current policies regarding air pollution control are mostly ineffective or applied in an unproductive manner. The air quality is a major issue for both, developed and developing countries. The World Health Organization [3] draws the attention about this global issue, especially in high urbanized regions with increased levels of traffic. Overall, in developing countries, the management practices to improve urban air quality are generally limited and the budget allocated for urban air quality management is also insufficient. However, exceptional cases can become a good practices example, like Tokyo, where air quality measurement capacity and management enabling capabilities are excellent [48]. Other significative countries are evaluated in [1] concerning urban air quality management system/process, from which we mention USA and European Union. USA has introduced the Clean Air Act (CAA) since 1963, setting responsibilities and relationships among federal, state, tribal, and local agencies. In Europe, the air quality management practices were introduced in 1980 with directive S0/779/EEC. However, the actual implementation of the newest Directive 2008/50/EC is left to the discretion of each state member. For example, a decrease in emission levels of $\mathrm{NO}_{\mathrm{x}}$ and $\mathrm{PM}_{2.5}$ from transport sector from 1990 to 2005 has resulted in a reduction in health impacts in the range of only $2.5 \%$ in Bulgaria to $25 \%$ in Luxembourg and Switzerland[49].

Dedicated platforms which are connected to local authorities are utterly needed so that the dedicated institution take actions, resulting a healthier urban environment.

\section{Conclusions}

The problem of urban air quality remains a sensitive subject, considering measuring techniques, modelling, forecasting, management and population health risks. New technologies of pollution monitoring, which have been simplified and can be accessible to the simple citizens, give the opportunity to raise awareness about clean environment, human health and civic implication.

This work was supported by the grants of the Romanian National Authority for Scientific Research, CNCSIS UEFISCDI, SMARTSENSE - Real time SMART application for urban air quality management respecting the SENSitivE categories of population, PN-III-P2-2.1-PED-2016-1285.

\section{References}

1. Gulia, S., et al., Urban air quality managementA review. Atmospheric Pollution Research, 2015. 6(2): p. 286-304.

2. OECD, The Economic Consequences of Outdoor Air Pollution. OECD Publishing.

3. WHO, Ambient air pollution: A global assessment of exposure and burden of disease, W.H. Organization, Editor. 2016.

4. WHO, Reducing global healh risks through mitigation of short-lived climate pollutants. Scoping report for policymakers, W.H. Organization, Editor. 2015.

5. WHO, Global surveillance, prevention and control of chronic respiratory diseases. A comprehensive approach, ed. W.H. Organization. 2007.

6. Diapouli, E., et al., Evolution of air pollution source contributions over one decade, derived by PM10 and PM2.5 source apportionment in two metropolitan urban areas in Greece. Atmospheric Environment, 2017. 164(Supplement C): p. 416-430.

7. Daher, N., et al., Characterization, sources and redox activity of fine and coarse particulate matter in Milan, Italy. Atmospheric Environment, 2012. 49(Supplement C): p. 130141 .

8. Agency, E.E., Air quality in Europe - 2017 report. 2017.

9. Kim, K.-H., E. Kabir, and S. Kabir, A review on the human health impact of airborne particulate matter. Environment international, 2015. 74: p. 136-143.

10. EPA, Clean Air Act Overview- Air Pollution: Current and Future Challenges, U.S.E.P. Agency, Editor. 1990.

11. ORGANIZATION, W.H., Indoor air pollutants:exposure and health effects, in Reports and Studies, EURO, Editor. 1982, WHO: Copenhagen. 
12. ORGANIZATION, W.H., Indoor air quality research, in Reports and Studies, EURO, Editor. 1984, WHO: Copenhagen.

13. Redlich, C.A., J. Sparer, and M.R. Cullen, Sickbuilding syndrome. The Lancet, 1997. 349(9057): p. 1013-6.

14. Zhong, J., X.-M. Cai, and W.J. Bloss, Coupling dynamics and chemistry in the air pollution modelling of street canyons: A review. Environmental Pollution, 2016. 214: p. 690704.

15. Peng, H., Air quality prediction by machine learning methods. 2015.

16. Adams, M.D. and P.S. Kanaroglou, Mapping real-time air pollution health risk for environmental management: Combining mobile and stationary air pollution monitoring with neural network models. Journal of Environmental Management, 2016. 168: p. 133141.

17. Hoek, G., et al., Estimation of long-term average exposure to outdoor air pollution for a cohort study on mortality. J Expo Anal Environ Epidemiol, 2001. 11(6): p. 459-69.

18. Vardoulakis, S., et al., Modelling air quality in street canyons: a review. Atmospheric environment, 2003. 37(2): p. 155-182.

19. Font, A., et al., Degradation in urban air quality from construction activity and increased traffic arising from a road widening scheme. Science of The Total Environment, 2014. 497-498: p. 123-132.

20. Atkinson, R., Atmospheric chemistry of VOCs and NO x. Atmospheric environment, 2000. 34(12): p. 2063-2101.

21. Svensson, U., PHOENICS in environmental flows. A review of applications at SMHI. Lecture Notes in Engineering, 1986. 18: p. 8796.

22. Baklanov, A., Application of CFD Methods for Modelling in Air Pollution Problems: Possibilities and Gaps. Environmental Monitoring and Assessment, 2000. 65(1): p. 181-189.

23. Chu, A.K.M., R.C.W. Kwok, and K.N. Yu, Study of pollution dispersion in urban areas using Computational Fluid Dynamics (CFD) and Geographic Information System (GIS). Environmental Modelling \& Software, 2005. 20(3): p. 273-277.

24. Neofytou, P., et al., Computational Fluid Dynamics Modelling of the Pollution Dispersion and Comparison with Measurements in a Street Canyon in Helsinki. Environmental Modeling \& Assessment, 2008. 13(3): p. 439448.

25. Haghighat, F. and P.A. Mirzaei, Impact of nonuniform urban surface temperature on pollution dispersion in urban areas. Building Simulation, 2011. 4(3): p. 227.

26. Jeanjean, A.P.R., et al., A CFD study on the effectiveness of trees to disperse road traffic emissions at a city scale. Atmospheric Environment, 2015. 120: p. 1-14.

27. McNabola, A., B.M. Broderick, and L.W. Gill, A numerical investigation of the impact of low boundary walls on pedestrian exposure to air pollutants in urban street canyons. Science of The Total Environment, 2009. 407(2): p. 760769.

28. Toja-Silva, F., et al., CFD simulation of CO2 dispersion from urban thermal power plant: Analysis of turbulent Schmidt number and comparison with Gaussian plume model and measurements. Journal of Wind Engineering and Industrial Aerodynamics, 2017. 169: p. 177-193.

29. Lal, B. and S.S. Tripathy, Prediction of dust concentration in open cast coal mine using artificial neural network. Atmospheric Pollution Research, 2012. 3(2): p. 211-218.

30. Nejadkoorki, F. and S. Baroutian, Forecasting Extreme PM10 Concentrations Using Artificial Neural Networks. International Journal of Environmental Research, 2012. 6(1): p. 277284.

31. Luecken, D.J., W.T. Hutzell, and G.L. Gipson, Development and analysis of air quality modeling simulations for hazardous air pollutants. Atmospheric Environment, 2006. 40(26): p. 5087-5096.

32. Kurt, A., et al., An online air pollution forecasting system using neural networks. Environment International, 2008. 34(5): p. 592598.

33. Wilson, R., Air Pollution, the Automobile, and Public Health. Environment: Science and Policy for Sustainable Development, 1989. 31(4): p. 25-27.

34. Solazzo, E., et al., Model evaluation and ensemble modelling of surface-level ozone in Europe and North America in the context of AQMEII. Atmospheric Environment, 2012. 53: p. 60-74.

35. Cartier, Y., T. Benmarhnia, and A. Brousselle, Tool for assessing health and equity impacts of interventions modifying air quality in urban environments. Evaluation and Program Planning, 2015. 53: p. 1-9.

36. Prasad, K., A.K. Gorai, and P. Goyal, Development of ANFIS models for air quality forecasting and input optimization for reducing the computational cost and time. Atmospheric Environment, 2016. 128: p. 246-262.

37. Borrego, C., et al., Urban scale air quality modelling using detailed traffic emissions estimates. Atmospheric Environment, 2016. 131: p. 341-351.

38. Bowman, K.W., Toward the next generation of air quality monitoring: Ozone. Atmospheric Environment, 2013. 80: p. 571-583.

39. Martin, R.V., Satellite remote sensing of surface air quality. Atmospheric Environment, 2008. 42(34): p. 7823-7843. 
40. $\mathrm{Hu}, \mathrm{D}$., et al., Urban air quality, meteorology and traffic linkages: Evidence from a sixteenday particulate matter pollution event in December 2015, Beijing. Journal of environmental sciences (China), 2017. 59: p. 30-38.

41. Rashid, B. and M.H. Rehmani, Applications of wireless sensor networks for urban areas: A survey. Journal of Network and Computer Applications, 2016. 60: p. 192-219.

42. Al-Ali, A.R., I. Zualkernan, and F. Aloul, A Mobile GPRS-Sensors Array for Air Pollution Monitoring. IEEE Sensors Journal, 2010. 10(10): p. 1666-1671.

43. Hubbell, B.J., et al., Understanding social and behavioral drivers and impacts of air quality sensor use. Science of The Total Environment, 2018. 621: p. 886-894.

44. Hasenfratz, D., et al., Participatory air pollution monitoring using smartphones. Mobile Sensing, 2012. 1: p. 1-5.

45. Sammarco, M., et al., Using geosocial search for urban air pollution monitoring. Pervasive and Mobile Computing, 2017. 35: p. 15-31.

46. Ahmed, A.A.N., et al., A Participatory Sensing Framework for Environment Pollution Monitoring and Management. arXiv preprint arXiv:1701.06429, 2017.

47. Sá, E., et al., Climate change and pollutant emissions impacts on air quality in 2050 over Portugal. Atmospheric Environment, 2016. 131: p. 209-224.

48. Santosa, S.J., T. Okuda, and S. Tanaka, Air Pollution and Urban Air Quality Management in Indonesia. CLEAN - Soil, Air, Water, 2008. 36(5-6): p. 466-475.

49. Pulles, M., Impact of selected policy measures on Europe's air quality. 2010. 